PROCEEDINGS OF THE

AMERICAN MATHEMATICAL SOCIETY

Volume 33, Number 1, May 1972

\title{
THE LATTICE OF IDEALS OF A COMPACT SEMILATTICE
}

\author{
A. R. STRALKA
}

\begin{abstract}
It is shown that, if $L$ is a compact distributive topological lattice with enough continuous join-preserving maps into $I$ to separate points, then there is a continuous lattice homcmorphism from $\mathscr{M}(L)$, the lattice of $M$-closed subsets of $L$, onto $L$. If $J(L)$, the set of join-irreducible elements of $L$, is a compact semilattice then $L$ is iseomorphic with. $\mathscr{H}(J(L))$.
\end{abstract}

I. Introduction. Let $S=(S, \wedge)$ be a compact, metric, topological semilattice and let $\mathscr{M}(S)$ be the set of all closed subsets $A$ of $S$ such that $A=A \wedge S$. In the sense of semigroup theory the elements of $\mathscr{M}(S)$ are ideals of $S$ while in the sense of lattice theory they are $M$-closed subsets of $S$. When $\mathscr{M}(S)$ is endowed with the Hausdorff metric and with operations $\vee$ and $\wedge$ defined to be set-theoretic union and intersection, respectively, $(\mathscr{M}(S), \vee, \wedge)$ becomes a compact, metric, distributive topological lattice, and moreover there is a natural imbedding $\rho_{S}$ of $S$ into $\mathscr{M}(S)$ defined by $\rho_{S}(x)=x \wedge S$ (cf. [5]). $\mathscr{M}(S$; provides a ready supply of nontrivial examples of compact, infinite-dimensional topological lattices. As such it has found use in [5] and [8].

In this paper we show that if $L$ is a compact topological lattice with enough continuous join-preserving maps into $I$ (the usual lattice on the closed real interval $[0,1])$ to separate points then there is a continuous join-preserving map $\Phi$ of $\mathscr{M}(L)$ onto $L$ and if $L$ is distributive then $\Phi$ is a lattice homomorphism. If in addition to being distributive $J(L)$, the set of join-irreducible elements of $L$, is a compact semilattice then $L$ is iseomorphic with $\mathscr{M}(J(L))$. This serves to characterize those lattices which can be the lattice of ideals of a compact semilattice.

We shall use the following definitions and notation. Let $L$ and $M$ be topological lattices. A point $x \in M$ is join-irreducible if $x=a \vee b$ implies that $x=a$ or $x=b$. The set of join-irreducible elements of $L$ will be denoted by $J(L)$ and if $A \subseteq L$ then $J(A)=J(L) \cap A . J P(L, M)$ is the set of continuous join-preserving maps of $L$ into $M ; M P(L, M)$ is the set of continuous

Received by the editors July 21, 1971.

AMS 1970 subject classifications. Primary 22A15, 06A20, 54F05.

Key words and phrases. Compact semilattice, lattice of ideals.

c) American Mathematical Society 1972 
meet-preserving maps of $L$ into $M$ (if $L$ and $M$ are only topological semilattices then $M P(L, M)$ will denote the set of continuous semilattice homomorphisms of $L$ into $M)$; and $\operatorname{Hom}(L, M)$ is the set of lattice homomorphisms of $L$ into $M$. For $q: L \rightarrow M, Z(q)=\{x \in L ; x$ is minima! in $\left.\varphi^{-1}(\gamma(x))\right\}$. $A^{*}$ will denete the topological closure of the set $A$ and $A^{\circ}$ is the interior of $A$.

2. $\mathscr{M}(S)$. We list several miscellaneous properties of $\mathscr{U}(S)$.

THEOREM 2.1. Let $S$ and $T$ be compact, metric, topological semilattices. Then

(1) $\rho_{S}(S)=J(\mathscr{H}(S))$

(2) If $\theta \in M P(S, T)$ then there is $\Phi \in \operatorname{Hom}(\mathscr{H}(S), \mathscr{U}(T))$ such that the following diagram commutes.

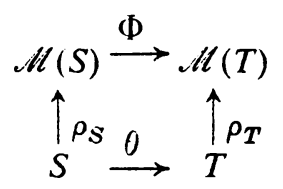

(3) $\operatorname{MP}(S, I)$ separates points if and only if $\operatorname{Hom}(\mathscr{U}(S), I)$ separates points.

Proof. (1) Suppose that $A, B \in \mathscr{M}(S)$ and $A \vee B=x \wedge S$. Then either $x \in A$ or $x \in B$. This implies that $x \wedge S \subseteq A$ or $x \wedge S \subseteq B$ which implies that $x \wedge S=A$ or $x \wedge S=B$. Hence $x \wedge S \in J(\mathscr{H}(S))$.

Conversely suppose that $A \in \mathscr{U}(S)$ and $A \notin \rho_{S}(S)$. Let $B$ be the set of maximal elements of $A$. Since $A \ddagger \rho_{S}(S), B$ is nondegenerate. Hence $B^{*}=B_{1} \cup B_{2}$ where $B_{1}$ and $B_{2}$ are closed proper subsets of $B^{*}$. Then $B_{1} \wedge S$ and $B_{2} \wedge S$ are members of $\mathscr{H}(S)$ and $\left(B_{1} \wedge S\right) \vee\left(B_{2} \wedge S\right)=B^{*} \wedge S=A$. Therefore $A \notin J(\mathcal{U}(S))$.

(2) From [3] or [6] we know that $\theta$ induces a continuous map $\Phi$ from $\mathscr{M}(S)$ into $\mathscr{M}(T)$ defined by $\Phi(A)=q(A) \wedge T$. Since unions are preserved by maps $\Phi$ is join-preserving. In general $\theta(A \wedge B) \subseteq \theta(A) \wedge \theta(B)$. Let $t \in \theta(A) \cap$ $\theta(B)$. Then there are $a \in A$ and $b \in B$ such that $\theta(a)=t=\theta(b) . a \wedge b \in A \wedge B=$ $A \cap B$. Hence $\theta(a \wedge b)=\theta(a) \wedge \theta(b)=t$.

(3) follows from (2) and the fact that $\mathscr{U}(I)$ is iseomorphic with $I$.

Part (3) below is slightly more general than the corollary to Lemma 4 of [7]; however the proof is about the same.

LEMMA 2.1. Let $L$ be a compact topological lattice.

(1) If $q \in J P(L, I)$ then $Z(q) \subseteq J(L)$.

(2) If $x \in L$ and $q \in J P(L, I)$ then there is $z \in Z(y)$ such that $\gamma(x)=q(z)$ and $x \geqq z$.

(3) If $x \in L$ and $J P(L, I)$ separates points then $x=\bigvee J(x \wedge L)$. 
Proof. (1) Let $z \in Z(\varphi)$ and suppose that $z=a \vee b$. Then $q(z)=$ $\varphi(a \vee b)=q(a) \vee q(b)$. Because $I$ is a chain $q(z)=\varphi(a)$ or $q(z)=q(b)$. We may take $q(z)=q(a)$. Then because $a \leqq z$ and $z \in Z(\varphi)$ we have $z=a$. Thus $z \in J(L)$.

(2) Obvious.

(3) Let $x \in L$. If $x=0$ then $J(x \wedge L)=\{0\}$ and $x=\bigvee J(x \wedge L)$. Now suppose that $x \neq 0$ and suppose that $x>y=\bigvee J(x \wedge L)$. Since $J P(L, i)$ separates points we may choose $\varphi \in J P(L, I)$ separating $x$ and $y$. From (2) there is $z \in Z(\varphi)$ such that $q(z)=q(x)$ and $z \leqq x$. Then $z \in J(x \wedge L)$ by (1). However, we then have $z \vee y>y=\bigvee J(x \wedge L)$. This is a contradiction. Hence $x=$ $\bigvee J(x \wedge L)$.

Semilattices whose topologies have neighborhood bases of semilattices have been studied by Lawson in [4]. There he called them semilattices with small semilattices.

THEOREM 2.2. Let L be a compact topological lattice. Then the following conditions are equivalent.

(1) JP(L,I) separates points.

(2) The topology for $L$ has a neighborhood base of $V$-semilattices.

(3) The map $\Phi: \mathscr{M}(L) \rightarrow L$ defined by $\Phi(A)=\bigvee A$ is a member of $J P(\mathscr{M}(L), L)$. And moreover if $L$ is distributive then $\Phi \in \operatorname{Hom}(\mathscr{M}(L), L)$.

Proof. The equivalence of (1) and (2) has been established by Lawson in [4]. It is easily verified that the topology of $\mathscr{M}(L)$ has a neighborhood base of $v$-semilattices. Hence if $\Phi \in J P(\mathscr{M}(L), L)$ then, as a consequence of Theorem 2.2 of [4], (2) must hold. Thus we need only show that the equivalent conditions (1) and (2) imply (3).

It is easily seen that $\Phi$ is an order-preserving map of $\mathscr{M}(L)$ onto $L$. Suppose that $\Phi$ is not continuous. Then there must be a sequence $\left\{A_{n}\right\}$ of elements of $\mathscr{M}(L)$ converging to $A_{0} \in \mathscr{M}(L)$ such that $\left\{\Phi\left(A_{n}\right)\right\}$ does not converge to $\Phi\left(A_{0}\right)$. For each nonnegative integer $i$ let $a_{i}=\Phi\left(A_{i}\right)$. We may suppose that $\left\{a_{n}\right\}$ converges to $a_{*} \neq a_{0}$. Let $b_{0} \in A_{0}$. Then there is a sequence $\left\{b_{n}\right\}$ converging to $b_{0}$ such that for each $n, b_{n} \in A_{n}$. Then since $a_{n} \geqq b_{n}$ we have $a_{*} \geqq b_{0}$. This implies that $a_{*}>a_{0}$. Since $\mathscr{M}(L)$ is a topological lattice $\left\{A_{n} \cup A_{0}\right\}$ converges to $A_{0}$ and since $\Phi$ is order preserving, $\Phi\left(A_{n} \cup A_{0}\right) \geqq$ $\Phi\left(A_{n}\right)$ for every positive integer $n$. Thus we may assume that $A_{n}=A_{0} \cup A_{n}$ for every $n$. Since $a_{0} \notin a_{*} \vee L$ there is an open neighborhood $U$ of $a_{0}$ such that $U^{*}$ does not meet $a_{*} \vee L$. Then because $L$ satisfies (2) there is a $\vee$ semilattice $U_{0}^{\prime} \subseteq U$ which is a neighborhood of $a_{0} .\left\{x \in L ; a_{0} \vee x \in U_{0}^{\circ}\right\}$ is open by continuity and it contains $A_{0}$. Hence $U_{0}^{*} \wedge L$ is a neighborhood of $A_{0}$. Let $t=\bigvee\left(U_{0}^{*} \wedge L\right)=\bigvee U_{0}^{*} . t \notin a_{*} \vee L$ because $U_{v}^{*} \subseteq U^{*}$ and $U^{*}$ does not meet $a_{*} \vee L$. Then because $\left\{A_{n}\right\}$ converges to $A_{0}$ there is a positive integer 
$N$ such that if $n \geqq N$ then $A_{n} \subseteq t \wedge L$. Thus $\Phi\left(A_{n}\right) \leqq t$. This implies that $\lim \Phi\left(A_{n}\right)=a_{*} \leqq t$ which is a contradiction. Therefore $\Phi$ is continuous.

We now show that $\Phi \in J P(\mathscr{H}(L), L)$. Let $A, B \in \mathscr{H}(L)$ with $a=\Phi(A)$, $b=\Phi(B)$ and $c=\Phi(A \cup B)$. Because $\Phi$ is order-preserving $a \vee b \leqq c$. Because $L$ is a compact metric lattice there is a countable set $\left\{c_{1}, c_{2}, \cdots\right\} \subseteq A \cup B$ such that $\bigvee_{i=1}^{\infty} c_{i}=c$. Define $a_{i}=\bigvee\left\{c_{j} ; j \leqq 1\right.$ and $\left.c_{j} \in A\right\}$ and $b_{i}=$ $\bigvee\left\{c_{j} ; j \leqq i\right.$ and $\left.c_{j} \in B\right\}$. Then $a_{i} \vee b_{i}=\bigvee_{j=1}^{i} c_{j}$. The sequences $\left\{a_{i}\right\}$ and $\left\{b_{i}\right\}$ are increasing. Since $A$ and $B$ are compact, $\left\{a_{i}\right\}$ converges to $\vec{a} \in A$ and $\left\{b_{i}\right\}$ converges to $\bar{b} \in B$. By continuity, $\left\{a_{i} \vee b_{i}\right\}$ converges to both $\bar{a} \vee \bar{b}$ and $c$. Thus $\bar{a} \vee \bar{b}=c$. Then because $\bar{a} \leqq a$ and $\bar{b} \leqq b$ we have $a \vee b=c$, i.e.. $\Phi \in$ $J P(\mathscr{H}(L), L)$.

We now suppose that $L$ is distributive. Let $\Phi(A)=a, \Phi(B)=b$ and $\Phi(A \wedge B)=d$. Because $\Phi$ is order-preserving $d \leqq a \wedge b$. Because $L$ is compact and metric there are countable subsets $\left\{a_{1}, a_{2}, \cdots\right\},\left\{b_{1}, b_{2}, \cdots\right\}$ of $A$ and $B$ respectively such that $\bigvee_{i=1}^{\infty} a_{i}=a$ and $\bigvee_{i=1}^{\infty} b_{i}=b$. Let $\bar{a}_{i}=a_{1} \vee a_{2} \vee \cdots \vee$ $a_{i}$ and $\bar{b}_{i}=b_{1} \vee b_{2} \vee \cdots \vee b_{i}$. The sequences $\left\{\bar{a}_{i}\right\}$ and $\left\{\bar{b}_{i}\right\}$ converge to $a$ and $b$ respectively. Because of continuity $\left\{\bar{a}_{i} \wedge \bar{b}_{i}\right\}$ converges to $a \wedge b$. However $\bar{a}_{i} \wedge \bar{b}_{i}=\left(a_{1} \vee a_{2} \vee \cdots \vee a_{i}\right) \wedge\left(b_{i} \vee b_{2} \vee \cdots \vee b_{i}\right)=\bigvee_{1 \leqq j ; k \leqq i}\left(a_{j} \wedge b_{k}\right)$. Each $a_{j} \wedge b_{k} \in$ $A \cap B$. So it follows that $\bar{a}_{i} \wedge \bar{b}_{i} \leqq d$ for all $i$. Thus $a \wedge b \leqq d$. Hence $a \wedge b=d$. Therefore $\Phi \in \operatorname{Hom}(\mathscr{M}(L), L)$.

The next lemma is a modification of Theorem 4.1 of [4].

LEMMA 2.2. Let $S$ be a compact, metric, topological semilattice whose topology has a neighborhood base of semilattices. If $A$ is a closed prime ideal of $S$ then there is $\gamma \in M P(S, I)$ such that $q(A) \cap \gamma(S \backslash A)=\varnothing$.

Proof. Because $A$ is a prime ideal we may assume that $A=\{0\}$, for otherwise we need only deal with the Rees-quotient $S / A$. The topology for $S$ has a neighborhood base of semilattices so for each $x \in S \backslash\{0\}$ there is $y_{x} \in S \backslash\{0\}$ such that $x \in M\left(y_{x}\right)^{\circ} .(M(y)=\{s \in S ; s \wedge y=y\}$.) The collection $\mathbb{Z}=$ $\left\{\boldsymbol{M}\left(y_{x}\right)^{\circ} ; x \in S\{\{0\}\}\right.$ is an open cover of $S \backslash\{0\}$. Since $S$ is a compact metric space $S \backslash\{0\}$ is a Lindelöf space. Hence $\# /$ has a countable subcover which we write as $\left\{M\left(y_{1}\right)^{\circ}, M\left(y_{2}\right)^{2}, \cdots\right\}$. For each positive integer $i$ define $\bar{y}_{i}=y_{1} \cdot l_{2} \cdots \cdots y_{i}$. Because $\{0\}$ is a prime ideal $\bar{y}_{i} \in S\{\{0\}$ for all $i$. No generality is lost by assuming that $S$ has an identity element, 1 . Select $i_{1}$ such that $l \in M\left(y_{i_{1}}\right)^{2}$. Select $i_{2}$ such that $y_{i_{1}} \in M\left(y_{i_{2}}\right)^{\circ}$. In general select $i_{,+1}$ so that $y_{i} \in M\left(y_{i_{j+1}}\right)^{\circ}$. Let $V^{\prime}\left(1 / 2^{j}\right)=S \backslash M\left(y_{i}\right)$. With these modifications proceed with the proof as given by Lawson in [4]. It must then follow that the member of $q \in M P(S, l)$ so defined possesses the additional property that $\phi^{-1}(q(0))=\{0\}$.

The next result provides additional properties of the map $\Phi$ defined in Theorem 2.2. 
THEOREM 2.3. Suppose that L is a compact, metric, distributive topological lattice such that the topology of L has a neighborhood base of V-semilattices. If $\Phi$ is the homomorphism as defined in Theorem 2.2 then:

(1) If $A$ is the minimal element of $\Phi^{-1}(\Phi(A))$ then $A=J(A) * \wedge=$ $(J(A) \wedge L)^{*}$.

(2) If $\Phi(A)=\Phi(B)$ for $A, B \in \mathscr{M}(L)$ then $J(A)=J(B)$.

Proof. (1) Let $a \in A$. From part (3) of Lemma 2.1, $a=\bigvee J(a \wedge L) \leqq$ $\bigvee J(A)$. This implies that $\bigvee A \leqq \bigvee J(A)$. However $J(A) \subseteq A$ so $\bigvee J(A)=$ $\bigvee A$.

(2) We may assume that $A=\wedge \Phi^{-1}(\Phi(A))$ and $B=L \wedge b \cup A$. Let $\Phi(A)=y$. Applying the dual version of Lemma 2.2 and noting that if $x \in J(L)$ then $x \vee L$ is a prime dual ideal of $L$ we obtain $\theta \in J P(L, I)$ such that $b$ is the unique minimal element of $\theta^{-1}(\theta(y))$. $A$ is compact so $\theta(A)$ is compact. Then since $l$ is a chain $\bigvee \theta(A) \in \theta(A)$. This implies that there is $a \in A$ such that $\theta(a)=\theta(y)$. Then from part (2) of Lemma 2.1 there is $z \in Z(\theta)$ such that $\theta(a)=\theta(z)$ and $z \leqq a$. However $b$ is the unique minimal element of $\theta^{-1}(\theta(y))$. Hence $b=z \leqq a$. Thus $b \in A \cap J(L)=J(A)$.

Finally we characterize those lattices which are the lattices of ideals of compact semilattices.

THEOREM 2.4. Let $L$ be a compact, metric, distributive topological lattice. Then $L$ is iseomorphic to $\mathscr{M}(S)$ for some compact semilattices $S$ if and only if

(a) the topology of $L$ has a neighborhood base of $V$-semilattices;

(b) $J(L)$ is a compact semilattice.

Proof. If $S$ is a compact semilattice then from Theorem $2.1, J(\mathscr{M}(S))$ is iseomorphic with $S$. Also it is easily shown that $\mathscr{M}(S)$ has a base of $\mathrm{V}$-semilattices.

Conversely since $J(L)$ is a compact, metric semilattice, $\mathscr{M}(J(L))$ is a compact distributive lattice. Let $\psi: \mathscr{M}(J(L)) \rightarrow \mathscr{M}(L)$ be defined by $\psi(A)=A \wedge L$. Since $L$ is metric from Proposition 8.6 of [2] we may assume that its metric is subinvariant with respect to $\wedge$, i.e., for $a, b, c \in L$, $d(a, b) \geqq d(a \wedge c, b \wedge c)$. This implies that for $A, B \in \mathscr{M}(J(L)), d(A, B) \geqq$ $d(A \wedge L, B \wedge L)$. Hence $\psi$ is a continuous map. It can be easily shown that $\psi$ is an injection. Thus we may conclude that $\psi$ is an imbedding of $\mathscr{M}(J(L))$ into $\mathscr{M}(L)$. Upon applying Theorem 2.3 we see that $\psi(\mathscr{M}(J(L)))$ meets each $\Phi$-equivalence class precisely once. Therefore $\Phi_{\circ} \psi$ is an iseomorphism from $\mathscr{M}(J(L))$ into $L$.

It should be noted that, in the above theorem, $L$ is iseomorphic to $\mathscr{M}(J(L))$ and that the map $\Phi: \mathscr{M}(L) \rightarrow L$ has full cross-section. Also the above theorem is similar in nature to Theorem 3 of Chapter 18 of [1]. 
REMARK. The author is grateful to James W. Stepp for informing him that with the exception of Lemma 2.2. all of the results of this paper can be obtained without the assumption of metrizability.

\section{REFERENCES}

1. G. Birkhoff, Lattice theory, 3rd ed., Amer. Math. Soc. Colloq. Publ., vol. 25, Amer. Math. Soc., Providence, R.I., 1967. MR 37 \#2638.

2. K. H. Hofmann and P. S. Mostert, Elements of compact semigroups, Merrill, Columbus, Ohio, 1966. MR 35 \#285.

3. K. Kuratowski, Topologie. Vol. 1, PWN, Warsaw, 1958; English transl., Academic Press, New York; PWN, Warsaw, 1966. MR 19, 873; MR 36 \#840.

4. J. D. Lawson, Topological semilattices with small semilattices, J. London Math. Soc. (2) 1 (1969), 719-724. MR 40 \#6516.

5. _ Lattices with no interval homomorphisms, Pacific J. Math. 32 (1970), 459465. MR 41 \#1019.

6. E. A. Michael, Topologies on space of subsets, Trans. Amer. Math. Soc. 71 (1951), 152-182. MR 13, 54.

7. Dona Papert Strauss, Topological lattices, Proc. London Math. Soc. 18 (1968), 217-230.

8. E. D. Shirley and A. R. Stralka, Homomorphisms on connected topological lattices, Duke Math. J. 38 (1971), 483-490.

Department of Mathematics, University of California, Riverside, California 92502 